\title{
The time course of erythrocyte membrane fatty acid concentrations during and after treatment of non-human primates with increasing doses of an omega-3 rich phospholipid preparation derived from krill-oil
}

\author{
Petter-Arnt Hals ${ }^{1 *}$ (D), Xiaoli Wang ${ }^{2}$, Fabiana Piscitelli ${ }^{3}$, Vincenzo Di Marzo ${ }^{3}$ and Yong-Fu Xiao ${ }^{2}$
}

\begin{abstract}
Background: A commonly used measure to reflect the intake of the long-chain omega-3 fatty acids EPA and DHA is the omega-3 index, defined as the sum of EPA + DHA as \% of total fatty acids in erythrocyte membrane. When the omega- 3 index changes it follows that the relative fractions of other fatty acids in the membrane are also changed. In the present study, increasing doses of a preparation of omega-3 rich phospholipids extracted from krill oil were administered orally to non-human primates for 12 weeks and the time course of EPA, DHA and 22 other fatty acids in erythrocytes was determined bi-weekly during treatment and for 8 weeks after cessation of treatment. Plasma concentrations of six endocannabinoid-type mediators being downstream metabolites of some fatty acids analyzed in erythrocytes were also determined.
\end{abstract}

Methods: Six diabetic, dyslipidemic non-human primates were included, three in a vehicle control group and three being treated with the omega-3 rich phospholipid preparation. The vehicle control and test items were given daily by gavage and the test item doses were 50,150 and $450 \mathrm{mg}$ phospholipids/kg/day. Each dose level was given for four weeks. Blood was sampled at baseline and thereafter bi-weekly. Fatty acids were determined in erythrocytes by methylation followed by gas-chromatography. Endocannabinoids and endocannabinoid-like mediators were analyzed in plasma by liquid chromatography-atmospheric pressure chemical ionization-mass spectrometry.

Results: The treatment resulted in a dose-related increase in the fraction of EPA and DHA in erythrocyte membranes and a dose-related decrease of other poly-unsaturated fatty acids, in particular omega- 6 polyunsaturated fatty acids. Erythrocyte concentrations of saturated fatty acids remained unchanged throughout the experiment. Plasma concentrations of endocannabinoids and endocannabinoid-like mediators changed accordingly as those being downstream arachidonic acid decreased, downstream of the saturated palmitic and oleic acids remained unchanged while a downstream EPA metabolite increased.

Conclusion: Increasing the omega-3 index by administering an omega-3 rich phospholipid extracted from krill oil did not alter the ratio of unsaturated vs. saturated fatty acids in the erythrocyte membranes but only the relative concentrations of unsaturated fatty acids, in particular unsaturated omega- 6 fatty acids. Concentrations of saturated fatty acids remained unchanged.

Keywords: Phospholipids, Long-chain omega-3 fatty acids, EPA, DHA, Omega-3 index, Endocannabinoids

\footnotetext{
* Correspondence: petter-arnt.hals@akerbiomarine.com

'Aker Biomarine Antarctic AS, Oksenoyveien 10, N-1366 Lysaker, Norway

Full list of author information is available at the end of the article
} 


\section{Background}

The long-chain omega-3 fatty acids (FAs) eicosapentaenoic acid (EPA; C20:5n3) and docosahexaenoic acid (DHA; C22:6n3) are only to a very little extent produced by the human body but high levels can be obtained if they are supplied via the diet. EPA and DHA are found in marine sources, and a diet rich in fatty fish like mackerel, salmon and anchovies will secure a high intake of these FAs. A large number of scientific publications over the last few decades have demonstrated numerous beneficial health effects of a high intake of these long-chain omega-3 FAs (for a review, see [1]) and this has led to a significant industry delivering dietary supplements containing one or both of these two FAs. The source of the omega-3 $\mathrm{s}$ in these products is for the most part fatty fish, but various crustacean species like krill [2] and copepods [3], as well as algae [4], are also utilized.

The omega- 3 index is often used to describe the levels of omega-3 FAs in the body. This index is defined as the percentage of EPA + DHA in erythrocyte membranes relative to the total amount of FAs in the membrane [5]. The analytical method commonly applied for the determination of the index in erythrocytes is gas chromatography following methylation of the fatty acid [6]. The index in humans is normally varying from about $2-3 \%$, indicating a low EPA and DHA intake, up to about $11-12 \%$ which indicates a high intake [5].

Since an increased intake of long-chain omega-3 FAs will increase the fraction of EPA and DHA in the erythrocyte membrane it follows that the relative content of all or some of the other fatty acids and, subsequently, of their bioactive derivatives, must decrease, with a potential biological impact possibly beyond the mere alteration of the physiological function of those fatty acids. In the present study, increasing doses of a preparation of omega- 3 rich phospholipids from krill oil was administered orally to non-human primates for a total of 12 weeks and the time course of the FA profiles in their erythrocytes was determined at baseline, during treatment and also after cessation of treatment. In addition to EPA and DHA, 22 FAs with an abundance varying from a fraction of a percent up to more than $20 \%$ at baseline were analyzed biweekly to investigate which of these were changed in the membranes when increasing amounts of EPA and DHA were administered. Furthermore, the concentrations of the two endocannabinoids, anandamide and 2arachidonoylglycerol (2-AG), and four acylethanolamide endocannabinoid-like mediators, i.e. EPA-ethanolamide, DHA-ethanolamide, oleoylethanolamide and palmitoylethanolamide, were determined in plasma bi-weekly. The two endocannabinoids are downstream metabolites of arachidonic acid while the four endocannabinoid-like compounds are the ethanolamides of EPA, DHA, oleic acid and palmitic acid, respectively, [7] and it was expected that their plasma concentrations would reflect changes in the abundance of their parent FA in the membranes (see [8] and [9] for examples of such effects of krill preparations in humans and mice, respectively). Endocannabinoids and long-chain acetylethanolamides are known to have marked biological effects and changing the concentrations of these might potentially affect physiological processes in several organ systems since the receptors of these lipid mediators are widely distributed in the whole organism [10].

The data reported in this article originate from a study which also investigated the effects of the phospholipid preparation on several parameters known to be risk factors for development of cardiovascular disease; serum cholesterols, apolipoproteins, triglycerides, inflammatory biomarkers and diabetes biomarkers. Selected safety parameters were also analyzed. The results from this part of the study are reported separately [11].

\section{Results}

The relative fraction of the two main omega- 3 fatty acids present in the phospholipid preparation administered to the non-human primates, EPA and DHA, increased dose-dependently in the treated animals (Fig. 1) while in the controls, only a minor change in the levels of these FAs was observed. For EPA, the levels in the treated animals increased from $<1 \%$ at baseline to a mean of $8 \%$ at the end of treatment while DHA levels increased from a mean of $6 \%$ up to a mean of $10 \%$. The omega- 3 index (Fig. 2) increased from a mean of $7.2 \%$ at baseline to $9.2 \%$ at 4 weeks, i.e. at the end of the treatment period with the lowest dose of $50 \mathrm{mg} / \mathrm{kg} /$ day, to $13.1 \%$ at 8 weeks which was after end of treatment with the intermediate dose of $150 \mathrm{mg} / \mathrm{kg} /$ day and finally to $18.3 \%$ at the end of treatment with the high dose of $450 \mathrm{mg} / \mathrm{kg} /$ day at 12 weeks. During the 12-weeks of treatment with the vehicle control substance, which contained no EPA and DHA, the mean omega-3 index in the control group remained essentially unchanged as it only was reduced from $6.0 \%$ at baseline to $5.8 \%$ (Fig. 2).

Following cessation of treatment, the membrane concentration of EPA and DHA in the treated group declined consistent with first-order elimination kinetics (Fig. 1). The omega-3 index was reduced from a mean of $18.3 \%$ at the end of treatment to a mean of $11.8 \%$ 2 weeks into the wash-out period, and 8 weeks after end of treatment the mean omega-3 index in the treated group was $8.8 \%$ (Fig. 2). As seen from the figure the two fatty acids that constitute the omega- 3 index, EPA and DHA, have different kinetics and the rate and extent of increase and decrease of the index are clearly affected differently by the two FAs. 


\section{Eicosapentaenoic acid (C20:5n3)}

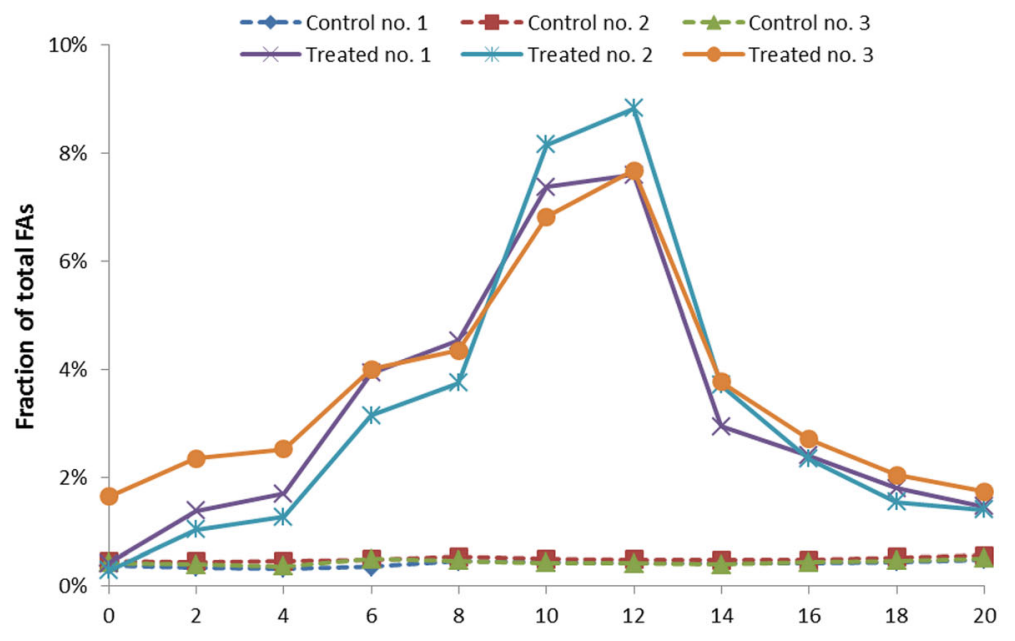

Docosahexaenoic acid (C22:6n3)

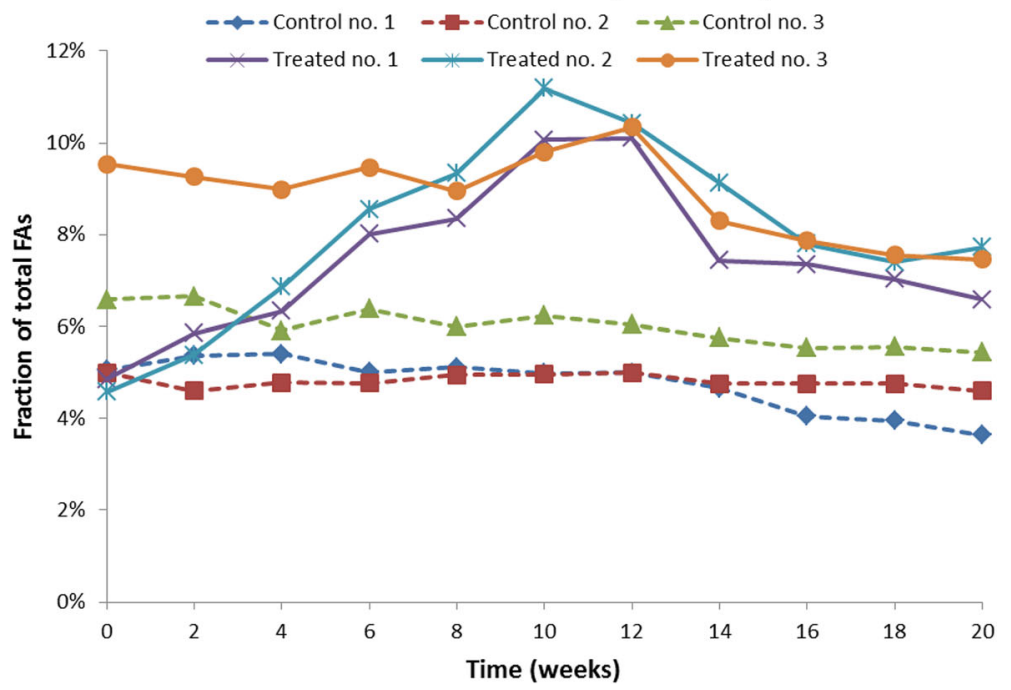

Fig. 1 Individual concentrations of the main omega-3 fatty acids, EPA and DHA, in erythrocyte membranes, given as \% of total fatty acids measured. Dotted lines represent control animals, solid lines represent treated animals which were given $50 \mathrm{mg} / \mathrm{kg} /$ day of the omega-3 rich phospholipids during week 1-4, $150 \mathrm{mg} / \mathrm{kg} /$ day during week 4-8 and $450 \mathrm{mg} / \mathrm{kg} /$ day during week 8-12. No treatment was given during week 12-20

The relative concentration of the omega- 3 variant of docosapentaenoic acid (DPA; C22:5n3), which may be considered a metabolite of EPA, was higher than for EPA at baseline; the mean was $2.8 \%$ and increased to a mean of $4.5 \%$ at the end of the 12 -week treatment (Fig. 5).

The mean fractions of 22 other fatty acids in erythrocyte membranes at each sampling time-point in the treated and control animals are presented in Table 1 . These data are further visualized in Figs. 3, 4 and 5. The erythrocyte membrane fraction of the saturated FAs was apparently unchanged when the omega-3 FAs increased. The less abundant myristic acid (C14:0), arachidic acid (C20:0), behenic acid (C22:0) and lignoceric acid (C24:0) showed no consistent trend of increase or decrease (Fig. 3). All these FAs were present at $<1 \%$ at baseline. The relative fraction of the major saturated FAs, palmitic acid (C16:0) and stearic acid (C18:0), which both were present in the membrane at a fraction of $>15 \%$ at baseline, was unchanged throughout the 20 weeks the study lasted (Table 1 and Fig. 5). On the other hand, the relative fractions of most of the unsaturated omega- 6 FAs were clearly altered during the study as the fraction of EPA and DHA increased, but with some exceptions.

A considerable reduction was seen for arachidonic acid (C20:4n6) which was reduced from a mean of 11.7 to $8.1 \%$ at 12 weeks, i.e. a reduction of approximately $30 \%$ from baseline (Fig. 5). During the 8-week wash-out 


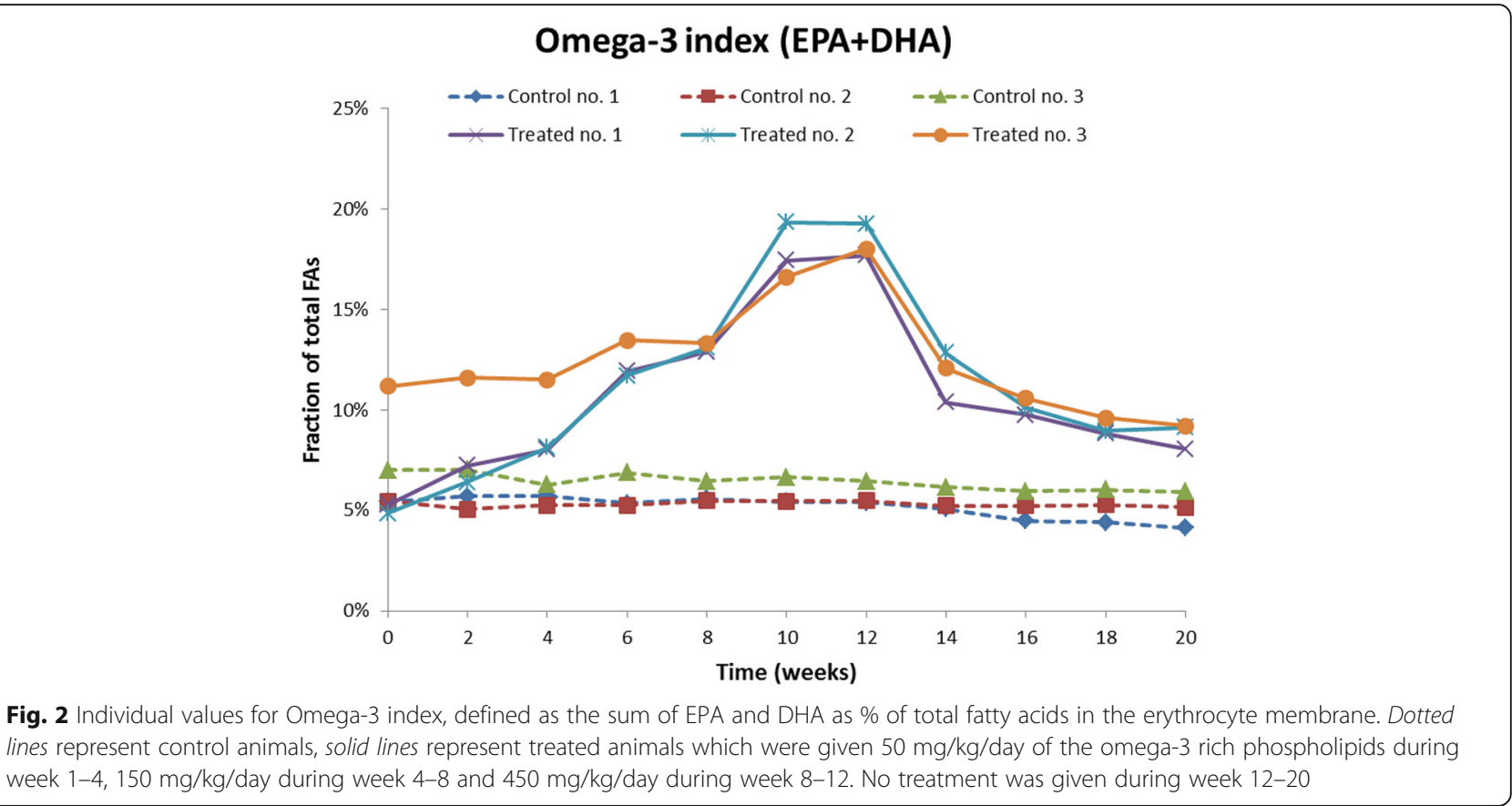

period a clear rebound effect was observed and at the end of this period, the arachidonic acid level was only $6 \%$ lower than at baseline. Another abundant unsaturated FA, linoleic acid (C18:2n6) with a baseline level of $22 \%$, was reduced down to $17.1 \%$ after the 12 week treatment (Fig. 5), a relative reduction of $27 \%$. Oleic acid $(\mathrm{C} 18: 1 \mathrm{n} 9)$ had a mean baseline level of $10.4 \%$ in the treated group and was reduced to a mean of $9.1 \%$ at the end of treatment (Fig. 5), which represents a relative reduction of about $12 \%$. The fraction of both these FAs approached their baseline levels during the 8 week wash-out period. Alpha-linolenic acid (C18:3n3) and gamma-linolenic acid (C18:3n6), both polyunsaturated FAs present at very low levels in the membranes, were both reduced during treatment with the long-chain omega-3 rich phospholipids (Fig. 3). For all FAs being reduced during treatment, the extent of reductions were apparently depending on the dosages of EPA and DHA and the reductions were always most prominent during weeks 8-12 of treatment where the highest dose of $450 \mathrm{mg}$ phospholipids $/ \mathrm{kg} /$ day was administered.

The two medium-abundant unsaturated omega-6 FAs, dihomo-g-linolenic acid (C20:3n6) and docosatetraenoic acid (C22:4n6), had the largest relative reduction of all FAs measured. The former FA was reduced by almost $60 \%$; from a mean fraction of $1.84 \%$ at baseline to $0.75 \%$ at 12 weeks, while the latter was reduced by $72 \%$, i.e. from a mean fraction of $1.39 \%$ at baseline to a mean of $0.39 \%$ at 12 weeks (Fig. 4).

Three trans-FAs were measured in the erythrocyte membranes, palmitelaidic acid $(\mathrm{C} 16: 1 \mathrm{n} 7 \mathrm{t})$, elaidic acid
(C18:1n9t) and linoelaidic acid (C18:2n6t). None of these were abundant in the membranes as all three had a relative fraction of around $0.2 \%$ at baseline, but they appeared unchanged throughout the 20 weeks the study lasted (Fig. 3).

Generally, for all FAs which changed their relative concentration in the erythrocyte membranes, a clear rebound effect was observed and most of them were close to the baseline levels 8 weeks after cessation of treatment with the omega-3 rich phospholipid preparation.

The results from the analyses of endocannabinoids and endocannabinoid-like compounds in plasma are summarized in Table 2. As indicated by the standard error of the means given in the table, the plasma concentrations of these compounds showed a marked variation between the animals and for some of the animals, including the controls, the concentrations also varied considerably from one time-point to the next. However, when adjusting the mean of the three animals in the treated group with the mean for the control group, the pattern for each of the endocannabinoid-type compounds in the treated group was generally consistent with the changes in the erythrocyte concentrations of the related FAs. Anandamide (arachidonoylethanolamide) and 2-AG are downstream derivatives of arachidonic acid and showed a decreasing trend in parallel with the decreased fraction of their parent FA in erythrocytes. EPA-ethanolamide, an endocannabinoid-like mediator ultimately formed from EPA, increased in parallel to the increased fraction of EPA in erythrocytes. DHA-ethanolamide, ultimately formed from DHA, 
Table 1 Mean fractions of fatty acids in erythrocyte membranes of the non-human primates ( $n=3$ in each of the two groups) at baseline, during and after treatment with the omega-3 rich phospholipid preparation, ranked according to increasing carbon chainlength and double-bonds

\begin{tabular}{|c|c|c|c|c|c|c|c|c|c|c|c|c|}
\hline \multirow[t]{2}{*}{ Fatty acid } & \multirow[t]{2}{*}{ Group } & \multirow[t]{2}{*}{ Baseline } & \multicolumn{10}{|c|}{ Week after start of treatment } \\
\hline & & & 2 & 4 & 6 & 8 & 10 & 12 & 14 & 16 & 18 & 20 \\
\hline \multirow[t]{2}{*}{ Myristic C14:0 } & Control & $0.18 \%$ & $0.16 \%$ & $0.18 \%$ & $0.19 \%$ & $0.16 \%$ & $0.13 \%$ & $0.17 \%$ & $0.17 \%$ & $0.16 \%$ & $0.14 \%$ & $0.17 \%$ \\
\hline & Treated & $0.23 \%$ & $0.22 \%$ & $0.22 \%$ & $0.19 \%$ & $0.21 \%$ & $0.21 \%$ & $0.20 \%$ & $0.15 \%$ & $0.21 \%$ & $0.19 \%$ & $0.20 \%$ \\
\hline \multirow[t]{2}{*}{ Palmitic C16:0 } & Control & $20.7 \%$ & $21.0 \%$ & $21.6 \%$ & $21.7 \%$ & $21.3 \%$ & $20.8 \%$ & $20.5 \%$ & $20.6 \%$ & $21.4 \%$ & $20.3 \%$ & $20.8 \%$ \\
\hline & Treated & $21.9 \%$ & $22.5 \%$ & $22.7 \%$ & $22.0 \%$ & $22.5 \%$ & $23.4 \%$ & $21.9 \%$ & $22.5 \%$ & $22.3 \%$ & $21.8 \%$ & $21.4 \%$ \\
\hline \multirow[t]{2}{*}{ Palmitoleic C16:1n7 } & Control & $0.15 \%$ & $0.14 \%$ & $0.16 \%$ & $0.15 \%$ & $0.14 \%$ & $0.14 \%$ & $0.15 \%$ & $0.14 \%$ & $0.13 \%$ & $0.13 \%$ & $0.13 \%$ \\
\hline & Treated & $0.27 \%$ & $0.28 \%$ & $0.28 \%$ & $0.22 \%$ & $0.20 \%$ & $0.15 \%$ & $0.15 \%$ & $0.19 \%$ & $0.23 \%$ & $0.22 \%$ & $0.22 \%$ \\
\hline \multirow[t]{2}{*}{ Palmitelaidic C16:1n7t } & Control & $0.07 \%$ & $0.07 \%$ & $0.07 \%$ & $0.07 \%$ & $0.08 \%$ & $0.06 \%$ & $0.08 \%$ & $0.07 \%$ & $0.07 \%$ & $0.06 \%$ & $0.07 \%$ \\
\hline & Treated & $0.12 \%$ & $0.14 \%$ & $0.14 \%$ & $0.14 \%$ & $0.15 \%$ & $0.16 \%$ & $0.17 \%$ & $0.12 \%$ & $0.13 \%$ & $0.12 \%$ & $0.12 \%$ \\
\hline \multirow[t]{2}{*}{ Stearic C18:0 } & Control & $18.1 \%$ & $18.1 \%$ & $18.1 \%$ & $18.3 \%$ & $18.0 \%$ & $17.6 \%$ & $17.8 \%$ & $18.1 \%$ & $18.0 \%$ & $17.8 \%$ & $17.9 \%$ \\
\hline & Treated & $15.9 \%$ & $16.1 \%$ & $16.0 \%$ & $16.0 \%$ & $16.4 \%$ & $15.2 \%$ & $15.7 \%$ & $16.5 \%$ & $16.2 \%$ & $16.0 \%$ & $16.0 \%$ \\
\hline \multirow[t]{2}{*}{ Oleic C18:1n9 } & Control & $9.54 \%$ & $9.49 \%$ & $9.73 \%$ & $9.72 \%$ & $9.63 \%$ & $9.50 \%$ & $9.49 \%$ & $9.59 \%$ & $9.52 \%$ & $9.30 \%$ & $9.34 \%$ \\
\hline & Treated & $10.4 \%$ & $10.2 \%$ & $9.98 \%$ & $9.43 \%$ & $9.45 \%$ & $8.88 \%$ & $9.14 \%$ & $9.80 \%$ & $9.73 \%$ & $9.72 \%$ & $9.46 \%$ \\
\hline \multirow[t]{2}{*}{ Elaidic C18:1n9t } & Control & $0.14 \%$ & $0.13 \%$ & $0.14 \%$ & $0.15 \%$ & $0.14 \%$ & $0.15 \%$ & $0.16 \%$ & $0.17 \%$ & $0.12 \%$ & $0.12 \%$ & $0.12 \%$ \\
\hline & Treated & $0.14 \%$ & $0.15 \%$ & $0.15 \%$ & $0.15 \%$ & $0.17 \%$ & $0.18 \%$ & $0.17 \%$ & $0.16 \%$ & $0.14 \%$ & $0.13 \%$ & $0.12 \%$ \\
\hline \multirow[t]{2}{*}{ Linoleic C18:2n6 } & Control & $22.7 \%$ & $22.1 \%$ & $22.7 \%$ & $22.5 \%$ & $22.4 \%$ & $23.3 \%$ & $23.4 \%$ & $23.6 \%$ & $24.8 \%$ & $25.4 \%$ & $25.4 \%$ \\
\hline & Treated & $22.4 \%$ & $20.3 \%$ & $20.8 \%$ & $19.0 \%$ & $18.9 \%$ & $16.7 \%$ & $17.1 \%$ & $21.2 \%$ & $23.4 \%$ & $23.9 \%$ & $24.1 \%$ \\
\hline \multirow[t]{2}{*}{ Linoelaidic C18:2n6t } & Control & $0.30 \%$ & $0.23 \%$ & $0.25 \%$ & $0.25 \%$ & $0.19 \%$ & $0.19 \%$ & $0.18 \%$ & $0.16 \%$ & $0.17 \%$ & $0.14 \%$ & $0.17 \%$ \\
\hline & Treated & $0.26 \%$ & $0.21 \%$ & $0.25 \%$ & $0.16 \%$ & $0.18 \%$ & $0.15 \%$ & $0.16 \%$ & $0.15 \%$ & $0.16 \%$ & $0.14 \%$ & $0.15 \%$ \\
\hline \multirow[t]{2}{*}{ alpha-linolenic C18:3n3 } & Control & $0.20 \%$ & $0.20 \%$ & $0.26 \%$ & $0.25 \%$ & $0.27 \%$ & $0.31 \%$ & $0.31 \%$ & $0.33 \%$ & $0.38 \%$ & $0.38 \%$ & $0.38 \%$ \\
\hline & Treated & $0.21 \%$ & $0.22 \%$ & $0.25 \%$ & $0.22 \%$ & $0.20 \%$ & $0.18 \%$ & $0.20 \%$ & $0.28 \%$ & $0.34 \%$ & $0.35 \%$ & $0.33 \%$ \\
\hline \multirow[t]{2}{*}{ gamma-linolenic C18:3n6 } & Control & $0.10 \%$ & $0.09 \%$ & $0.09 \%$ & $0.07 \%$ & $0.10 \%$ & $0.11 \%$ & $0.10 \%$ & $0.11 \%$ & $0.10 \%$ & $0.08 \%$ & $0.09 \%$ \\
\hline & Treated & $0.09 \%$ & $0.09 \%$ & $0.09 \%$ & $0.07 \%$ & $0.07 \%$ & $0.04 \%$ & $0.05 \%$ & $0.06 \%$ & $0.08 \%$ & $0.07 \%$ & $0.09 \%$ \\
\hline \multirow[t]{2}{*}{ Arachidic C20:0 } & Control & $0.35 \%$ & $0.35 \%$ & $0.35 \%$ & $0.35 \%$ & $0.37 \%$ & $0.35 \%$ & $0.36 \%$ & $0.37 \%$ & $0.35 \%$ & $0.37 \%$ & $0.38 \%$ \\
\hline & Treated & $0.32 \%$ & $0.31 \%$ & $0.33 \%$ & $0.32 \%$ & $0.34 \%$ & $0.32 \%$ & $0.32 \%$ & $0.33 \%$ & $0.34 \%$ & $0.34 \%$ & $0.37 \%$ \\
\hline \multirow[t]{2}{*}{ Eicosenoic C20:1n9 } & Control & $0.52 \%$ & $0.49 \%$ & $0.51 \%$ & $0.49 \%$ & $0.50 \%$ & $0.47 \%$ & $0.45 \%$ & $0.45 \%$ & $0.44 \%$ & $0.43 \%$ & $0.45 \%$ \\
\hline & Treated & $0.59 \%$ & $0.55 \%$ & $0.55 \%$ & $0.51 \%$ & $0.52 \%$ & $0.44 \%$ & $0.44 \%$ & $0.46 \%$ & $0.46 \%$ & $0.45 \%$ & $0.49 \%$ \\
\hline \multirow[t]{2}{*}{ Eicosadienoic C20:2n6 } & Control & $0.79 \%$ & $0.73 \%$ & $0.70 \%$ & $0.69 \%$ & $0.68 \%$ & $0.73 \%$ & $0.73 \%$ & $0.72 \%$ & $0.75 \%$ & $0.74 \%$ & $0.74 \%$ \\
\hline & Treated & $0.74 \%$ & $0.72 \%$ & $0.71 \%$ & $0.64 \%$ & $0.62 \%$ & $0.55 \%$ & $0.52 \%$ & $0.69 \%$ & $0.70 \%$ & $0.72 \%$ & $0.74 \%$ \\
\hline \multirow[t]{2}{*}{ Dihomo-g-linolenic C20:3n6 } & Control & $1.27 \%$ & $1.22 \%$ & $1.23 \%$ & $1.21 \%$ & $1.13 \%$ & $1.22 \%$ & $1.23 \%$ & $1.23 \%$ & $1.20 \%$ & $1.10 \%$ & $1.12 \%$ \\
\hline & Treated & $1.84 \%$ & $1.61 \%$ & $1.53 \%$ & $1.15 \%$ & $1.06 \%$ & $0.81 \%$ & $0.75 \%$ & $1.12 \%$ & $1.28 \%$ & $1.36 \%$ & $1.46 \%$ \\
\hline \multirow[t]{2}{*}{ Arachidonic C20:4n6 } & Control & $13.9 \%$ & $14.3 \%$ & $13.4 \%$ & $13.4 \%$ & $13.7 \%$ & $13.8 \%$ & $13.9 \%$ & $13.6 \%$ & $12.8 \%$ & $13.0 \%$ & $12.6 \%$ \\
\hline & Treated & $11.7 \%$ & $11.9 \%$ & $10.8 \%$ & $10.5 \%$ & $9.41 \%$ & $8.49 \%$ & $8.10 \%$ & $8.63 \%$ & $8.63 \%$ & $9.53 \%$ & $9.88 \%$ \\
\hline \multirow[t]{2}{*}{ Behenic C22:0 } & Control & $0.40 \%$ & $0.43 \%$ & $0.36 \%$ & $0.35 \%$ & $0.45 \%$ & $0.43 \%$ & $0.41 \%$ & $0.43 \%$ & $0.36 \%$ & $0.46 \%$ & $0.40 \%$ \\
\hline & Treated & $0.40 \%$ & $0.38 \%$ & $0.36 \%$ & $0.42 \%$ & $0.41 \%$ & $0.37 \%$ & $0.41 \%$ & $0.38 \%$ & $0.37 \%$ & $0.39 \%$ & $0.41 \%$ \\
\hline Docosatetraenoic C22:4n6 & Control & $1.33 \%$ & $1.33 \%$ & $1.32 \%$ & $1.31 \%$ & $1.33 \%$ & $1.34 \%$ & $1.40 \%$ & $1.36 \%$ & $1.29 \%$ & $1.30 \%$ & $1.29 \%$ \\
\hline & Treated & $1.39 \%$ & $1.24 \%$ & $1.11 \%$ & $0.87 \%$ & $0.70 \%$ & $0.49 \%$ & $0.39 \%$ & $0.42 \%$ & $0.47 \%$ & $0.64 \%$ & $0.74 \%$ \\
\hline Docosapentaenoic C22:5n3 & Control & $2.10 \%$ & $2.07 \%$ & $2.09 \%$ & $2.13 \%$ & $2.13 \%$ & $2.14 \%$ & $2.12 \%$ & $2.04 \%$ & $1.95 \%$ & $2.02 \%$ & $2.06 \%$ \\
\hline & Treated & $2.75 \%$ & $3.18 \%$ & $3.42 \%$ & $3.93 \%$ & $4.07 \%$ & $4.36 \%$ & $4.53 \%$ & $4.16 \%$ & $3.74 \%$ & $3.65 \%$ & $3.63 \%$ \\
\hline Docosapentaenoic C22:5n6 & Control & $0.40 \%$ & $0.54 \%$ & $0.34 \%$ & $0.36 \%$ & $0.49 \%$ & $0.48 \%$ & $0.39 \%$ & $0.41 \%$ & $0.27 \%$ & $0.42 \%$ & $0.40 \%$ \\
\hline & Treated & $0.41 \%$ & $0.38 \%$ & $0.30 \%$ & $0.48 \%$ & $0.43 \%$ & $0.38 \%$ & $0.39 \%$ & $0.31 \%$ & $0.29 \%$ & $0.41 \%$ & $0.46 \%$ \\
\hline Lignoceric C24:0 & Control & $0.40 \%$ & $0.46 \%$ & $0.33 \%$ & $0.30 \%$ & $0.48 \%$ & $0.47 \%$ & $0.41 \%$ & $0.39 \%$ & $0.31 \%$ & $0.46 \%$ & $0.39 \%$ \\
\hline & Treated & $0.39 \%$ & $0.35 \%$ & $0.32 \%$ & $0.48 \%$ & $0.44 \%$ & $0.39 \%$ & $0.44 \%$ & $0.35 \%$ & $0.32 \%$ & $0.41 \%$ & $0.44 \%$ \\
\hline
\end{tabular}


Table 1 Mean fractions of fatty acids in erythrocyte membranes of the non-human primates ( $n=3$ in each of the two groups) at baseline, during and after treatment with the omega-3 rich phospholipid preparation, ranked according to increasing carbon chainlength and double-bonds (Continued)

\begin{tabular}{|c|c|c|c|c|c|c|c|c|c|c|c|c|}
\hline \multirow[t]{2}{*}{ Nervonic C24:1n9 } & Control & $0.41 \%$ & $0.50 \%$ & $0.30 \%$ & $0.26 \%$ & $0.48 \%$ & $0.45 \%$ & $0.39 \%$ & $0.38 \%$ & $0.25 \%$ & $0.45 \%$ & $0.33 \%$ \\
\hline & Treated & $0.46 \%$ & $0.44 \%$ & $0.33 \%$ & $0.58 \%$ & $0.48 \%$ & $0.40 \%$ & $0.46 \%$ & $0.33 \%$ & $0.30 \%$ & $0.42 \%$ & $0.45 \%$ \\
\hline \multicolumn{3}{|c|}{ Dose (mg phospholipids/kg bw/day) } & \multicolumn{2}{|l|}{50} & \multicolumn{2}{|l|}{150} & \multicolumn{2}{|l|}{450} & \multicolumn{4}{|l|}{0} \\
\hline \multicolumn{3}{|c|}{ Dose (mg [EPA/DHA]/kg bw/day) } & \multicolumn{2}{|c|}{$9.35 / 5.48$} & \multicolumn{2}{|c|}{ 28.0/16.4 } & \multicolumn{2}{|c|}{$84.1 / 49.3$} & \multicolumn{4}{|l|}{$0 / 0$} \\
\hline
\end{tabular}

Doses of administered phospholipids and of EPA and DHA are shown at the end of the table

showed, however, no consistent trend of increase or decrease despite the observed increase in the fraction of DHA in the erythrocyte membranes. Two other endocannabinoid-like compounds, palmitoylethanolamide and oleoylethanolamide, ultimately formed from the saturated FAs palmitic acid and oleic acid, respectively, did not show any trend of change in the plasma concentration which is in line with the unchanged erythrocyte fraction of their parent FAs. Similar to the FAs in the erythrocyte membranes, the plasma concentration of the affected FA metabolites returned towards the baseline or even beyond the baseline levels after cessation of treatment with the phospholipid preparation.

\section{Discussion}

The main finding of the present investigation is that when the fractions of the long-chain omega-3 FAs EPA and DHA in erythrocyte membranes increased as a result of oral treatment with an omega-3 rich phospholipid preparation extracted from krill oil, the FAs reduced were only other unsaturated FAs and in particular omega- 6 FAs. None of the saturated FAs changed their relative presence in the membranes, whether they were abundant or not. The relative fraction of monounsaturated FAs seems to be less affected than polyunsaturated ones. Also the measured trans FAs, although present only in very low fractions, appeared unchanged during the treatment period. It is of note that the relative fractions of EPA and DHA increased markedly as a result of treatment, and the omega- 3 index, representing the sum of EPA and DHA in the membranes, increased from $7.3 \%$ at baseline to $18.2 \%$ at the end of the 12 -week treatment. The omega-3 dose-levels applied in this study are relevant in a human setting since the dose-levels of EPA + DHA used, when converted to human equivalent dose (HED) levels, amount to 300, 860 and $2580 \mathrm{mg} /$ day to an adult human of $60 \mathrm{~kg}$. The HED is calculated based on body surface area and obtained by dividing the dose in the monkeys, given as $\mathrm{mg} / \mathrm{kg}$, by 3.1 and multiplying by the human body weight [12].

Our data are in line with the results from the study by Katan et al. [13], who gave three doses of fish oil to adults and measured omega- 3 fatty acids as well as two omega- 6 fatty acids, linoleic and arachidonic acids, in erythrocytes. The omega- 3 fatty acids increased dosedependently while the two omega- 6 fatty acids were reduced dose-dependently. These authors did not report concentrations of any saturated fatty acids. However Ramprasath and co-workers compared the increase in omega-3 index, omega-6:omega-3 ratio, total saturated fatty acids, total monounsaturated fatty acids and total omega- 6 fatty acids after krill oil and fish oil after 4 weeks of treatment and found that total saturated fatty acids were unchanged despite a clear increase in omega- 3 index and a reduction in the omega-6:omega-3 ratio. These authors did not specify the levels of individual fatty acids except for EPA, DHA and DPA [14].

The potential consequences of these changes in the composition of the cell membranes induced by the administration of EPA and DHA were not investigated in this study but the assumption is that the balance between saturated and unsaturated FAs has implications for membrane fluidity and thereby cellular function [15]. Therefore, the observation that the membrane content of saturated vs. unsaturated FAs is relatively unaffected by the introduction of EPA and DHA is interesting. Of note are also the changes in the plasma concentrations of endocannabinoids and endocannabinoid-like mediators, which generally followed the changes in erythrocyte FA abundance. This was not surprising since these mediators are ultimately derived from membrane phospholipids containing the corresponding fatty acids in either the $s n-1$ (in the case of acylethanolamides) or $s n-2$ (in the case of 2-acylglycerols such as 2-AG) positions. Due to the various physiological effects of the endocannabinoids, in particular their regulatory effects on appetite, food intake, adipose tissue and ectopic fat accumulation and insulin sensitivity [16], a reduction of the relative abundance of their precursors and, subsequently, of their biosynthesized amounts, may have significant biological implications. Thus, the trend for the reduction in arachidonoylethanolamide (anandamide) and 2-AG levels may explain in part some of the beneficial metabolic effects observed with the krill oil preparation [11]. However, also EPA and DHA-derived ethanolamides are emerging as bona fide non-endocannabinoid mediators with potential anti-inflammatory actions [17] and we found here 

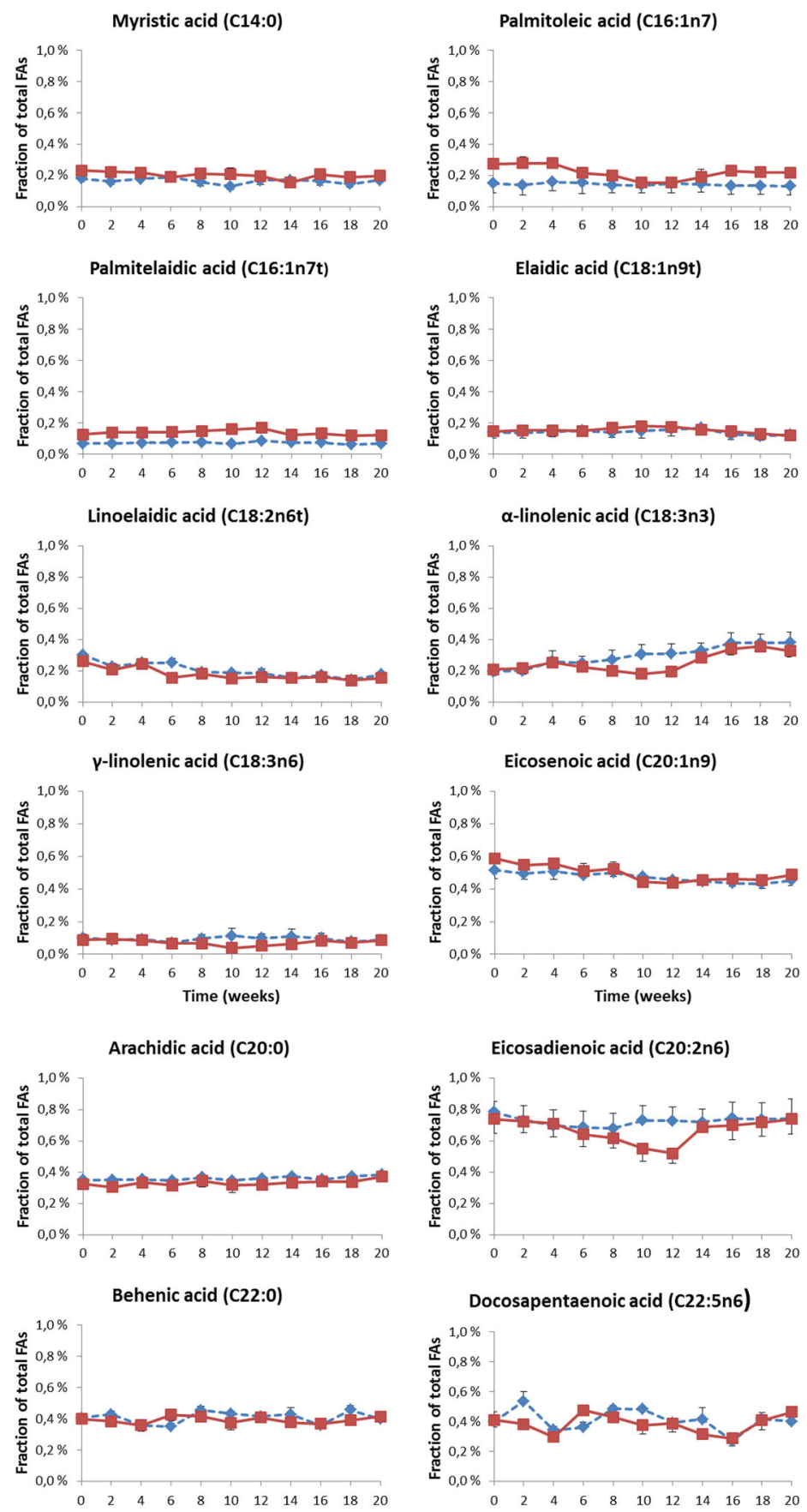

$$
\text { Docosapentaenoic acid (C22:5n6) }
$$
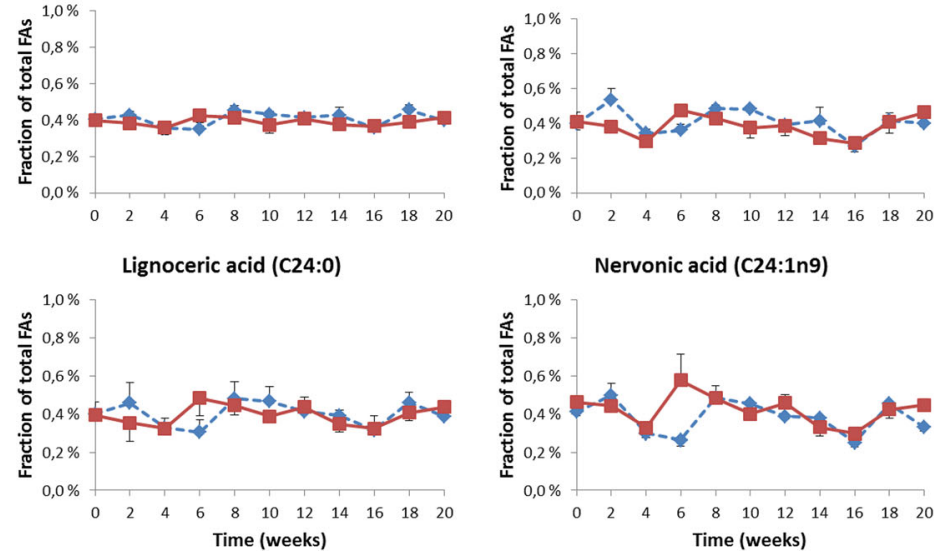

Fig. 3 (See legend on next page.) 
(See figure on previous page.)

Fig. 3 Mean concentrations and SEM of the less abundant ( $<1 \%$ at study start) fatty acids in erythrocyte membranes, given as \% of total fatty acids measured, shown in the order of increasing chain length. Blue dotted line represents the control group, red solid line represents the treated group which was given $50 \mathrm{mg} / \mathrm{kg} /$ day of the omega-3 rich phospholipids during week 1-4, $150 \mathrm{mg} / \mathrm{kg} /$ day during week 4-8 and $450 \mathrm{mg} / \mathrm{kg} / \mathrm{day}$ during week 8-12. No treatment was given during week 12-20

that EPA-ethanolamide, which is ultimately derived from the long chain omega-3 FA whose levels increased the most and most consistently in our study, i.e. EPA, was also increased following treatment with krill oil phospholipids.

The finding that the main effect of a high intake of omega-3 FAs is a progressive change in the relative abundance of the polyunsaturated FAs and not a displacement of saturated FAs from the cell membrane is also supporting the theory that the reported anti-inflammatory effects of omega-3 FAs may, at least partly, be due to a reduced membrane concentration of arachidonic acid which in turn should lead to reduced production of proinflammatory arachidonic acid metabolites like prostaglandins, leukotriens and thromboxanes [18]. Our data show that the fraction of arachidonic acid in the membranes was reduced by about $30 \%$ as a result of the 12-week treatment with increasing doses of the omega-3 rich phospholipids. An additional aspect is the effects of the so-called 'specialized proresolving mediators'; the resolvins, protectins and maresins, all molecules biosynthesized from long-chain omega-3 FAs and shown to stimulate proresolving cellular processes such as inhibition of neutrophil infiltration, enhancement of macrophage phagocytosis of bacteria and efferocytosis of cellular debris, and reduction of inflammatory pain through specific G-protein coupled receptors [19]. The proresolving effects of these specialized proresolving mediators would be expected to work in concert with the decreased proinflammatory effects caused by a reduced production of arachidonic acid metabolites and together, these two mechanisms might to a large extent explain the beneficial effects the long-chain omega-3 FAs have on inflammation.
The long-chain omega-3 FAs used in the present study were administered to the animals as phospholipids. The most common form of omega-3 FAs in the human diet is, however, triglycerides since omega-3 FAs in fish are mostly in this chemical form [2]. Our study did not compare the outcome of administration of omega-3 FAs as phospholipids and as triglycerides and the results obtained in our study should therefore not be generalized as effects of any omega-3 preparation used. Since omega-3 FAs in cell membranes mostly are incorporated in phospholipids, it might be that the chemical form administered has implications on how the FAs are distributed in cell membranes. There has been some debate in the literature whether delivery of long-chain omega-3 FAs as phospholipids, which is the case when the source is krill oil, is more efficient for building up the omega-3 index than when delivering these FAs as triglycerides like in most fish oils. A recent article by Yurko-Mauro and co-workers concluded that giving EPA and DHA in either ethyl ester, triglyceride or phospholipid form for 4 weeks created similar plasma and erythrocyte levels of the FAs [20]. However, other literature has indicated a more efficient uptake of these omega-3 FAs when given as krill oil than with fish oil $[21,22]$, resulting in higher omega-3 indices after dose-corrections. From a general point of view one would expect FAs to be absorbed almost completely from forms that are natural, i.e. triglycerides and phospholipids, since the digestion of fat in the gastrointestinal tract is tailor-made for optimal absorbtion of this type of nutrients [23]. It is of note, however, that Ramprasath and coworkers have published their observation that the content of phospholipids in krill oil may have an impact on the bioavailability of the omega-3 FAs [24]. Given that the preparation tested in
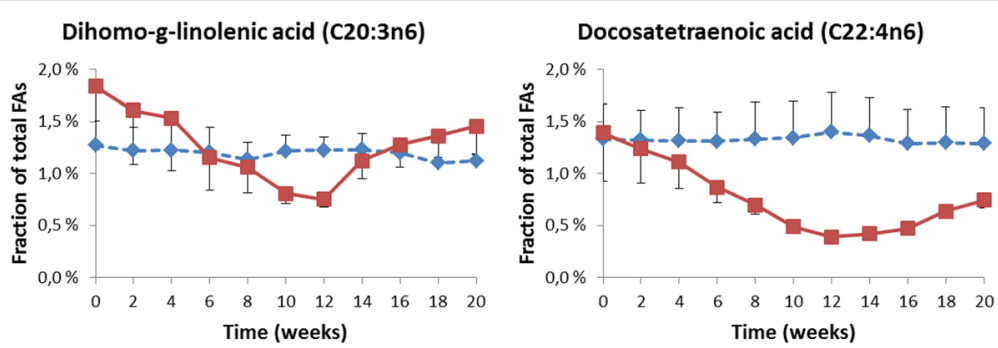

Fig. 4 Mean concentrations and SEM of the median abundant ( $>1 \%$ but $<2 \%$ at study start) fatty acids in erythrocyte membranes, given as $\%$ of total fatty acids measured, shown in the order of increasing chain length. Blue dotted line represents the control group, red solid line represents the treated group which was given $50 \mathrm{mg} / \mathrm{kg} /$ day of the omega-3 rich phospholipids during week 1-4, $150 \mathrm{mg} / \mathrm{kg} /$ day during week $4-8$ and $450 \mathrm{mg} / \mathrm{kg} /$ day during week 8-12. No treatment was given during week 12-20 


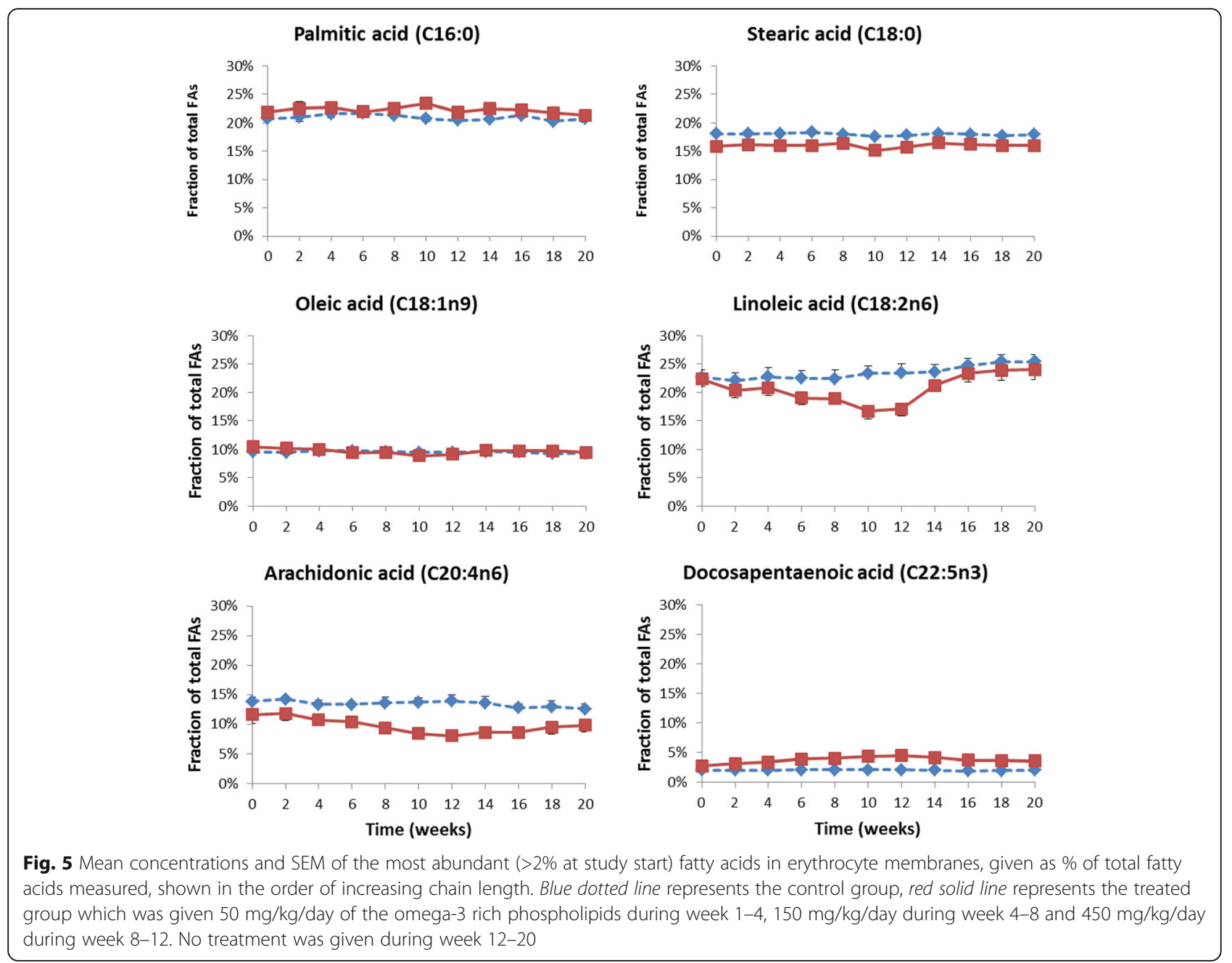

our study was highly purified with a phospholipid content of $>98 \%$, this may have led to a higher increase in the omega-3 index than would a normal krill-oil preparation with an approximately $40 \%$ phospholipid content, provided comparable doses of omega-3 FAs.

The omega-3 index determined in erythrocyte membranes is well correlated with omega-3 levels in other tissues, in particular heart tissue [25]. A high omega-3 index, preferably above $8 \%$, has been shown to be strongly correlated with a reduced risk of sudden cardiac death [26]. Furthermore, a low omega-3 index has also been shown to correlate with an increased risk for disease of the central nervous system like depression [27] and an increased intake of long-chain omega-3 FAs has been suggested as a treatment strategy for maintaining cognitive health into older age [28].

An obvious limitation of our study is the relatively low number of animals included in each of the control and treated groups, which precludes a statistical analysis of the data. However, given that the species used is a nonhuman primate, animal ethics suggests that the number used should be as low as possible, provided meaningful data can be obtained. Animals fed a standard diet over long periods of time, like those in the present study, will generally have much less variation in their baseline FA profiles than humans who have a variable diet and therefore more heterogeneous FA profiles. This was confirmed by the low variation in erythrocyte FA abundance between the animals in the control group. Also the consistent changes in the FAs in the treated animals indicate that the trends observed are reliable.

\section{Conclusions}

Feeding non-human primates with increasing doses of an omega-3 rich phospholipid preparation extracted from krill oil caused a dose-related increase in the fraction of the long-chain omega-3 FAs EPA and DHA in erythrocyte membranes and a dose-related decrease of other polyunsaturated FAs, and in particular omega-6 polyunsaturated FAs. Erythrocyte concentrations of saturated fatty acids remained unchanged throughout the experimental period. Hence, increasing the omega-3 index 
Table 2 Concentrations ( $\mathrm{pmol} / \mathrm{ml}$ ) of the endocannabinoid-type compounds measured in plasma from the non-human primates ( $n=3$ in each of the two groups) at baseline, during and after treatment with increasing doses of the omega-3 rich phospholipid preparation

\begin{tabular}{|c|c|c|c|c|c|c|c|c|c|c|c|c|c|}
\hline \multirow{2}{*}{$\begin{array}{l}\text { Endocannabinoid-type } \\
\text { compound }\end{array}$} & \multirow[t]{2}{*}{ Group } & \multirow[t]{2}{*}{ Value } & \multirow[t]{2}{*}{ Baseline } & \multicolumn{10}{|c|}{ Week after start of treatment } \\
\hline & & & & 2 & 4 & 6 & 8 & 10 & 12 & 14 & 16 & 18 & 20 \\
\hline \multirow[t]{4}{*}{ Anandamide } & \multirow[t]{2}{*}{ Control } & Mean & 4.24 & 4.80 & 5.60 & 4.31 & 4.82 & 4.66 & 6.02 & 6.19 & 2.82 & 5.19 & 5.15 \\
\hline & & SEM & 2.00 & 1.18 & 2.17 & 0.93 & 0.80 & 0.91 & 3.84 & 2.65 & 0.77 & 1.38 & 1.67 \\
\hline & \multirow[t]{2}{*}{ Treated } & Mean & 3.07 & 3.40 & 2.86 & 2.98 & 2.86 & 3.34 & 2.28 & 3.64 & 4.25 & 4.05 & 4.44 \\
\hline & & SEM & 0.52 & 1.54 & 0.93 & 0.56 & 0.92 & 1.35 & 0.97 & 1.35 & 1.69 & 1.53 & 0.43 \\
\hline \multirow[t]{4}{*}{ 2-arachidonoylglycerol } & \multirow[t]{2}{*}{ Control } & Mean & 14.3 & 21.0 & 23.1 & 21.9 & 27.8 & 20.7 & 28.6 & 22.7 & 19.0 & 18.6 & 22.4 \\
\hline & & SEM & 5.5 & 13.8 & 15.9 & 11.1 & 19.2 & 15.2 & 20.6 & 12.3 & 11.8 & 11.3 & 15.5 \\
\hline & \multirow[t]{2}{*}{ Treated } & Mean & 16.8 & 19.2 & 13.3 & 9.14 & 11.7 & 4.42 & 8.48 & 15.0 & 26.5 & 16.5 & 26.4 \\
\hline & & SEM & 6.4 & 9.2 & 7.9 & 1.12 & 3.3 & 1.60 & 4.20 & 10.0 & 19.1 & 9.1 & 11.3 \\
\hline \multirow[t]{4}{*}{ EPA ethanolamide } & \multirow[t]{2}{*}{ Control } & Mean & 1.36 & 1.45 & 0.91 & 1.25 & 0.22 & 0.57 & 1.00 & 1.07 & 2.07 & 2.04 & 2.84 \\
\hline & & SEM & 0.49 & 0.29 & 0.16 & 0.31 & 0.07 & 0.20 & 0.07 & 0.71 & 0.84 & 0.62 & 1.06 \\
\hline & \multirow[t]{2}{*}{ Treated } & Mean & 0.47 & 1.04 & 1.05 & 1.13 & 0.85 & 1.00 & 1.07 & 0.58 & 1.34 & 1.55 & 0.63 \\
\hline & & SEM & 0.13 & 0.48 & 0.34 & 0.26 & 0.24 & 0.17 & 0.26 & 0.22 & 0.93 & 0.51 & 0.35 \\
\hline \multirow[t]{4}{*}{ DHA ethanolamide } & \multirow[t]{2}{*}{ Control } & Mean & 1.23 & 4.67 & 5.17 & 6.64 & 4.95 & 4.63 & 4.61 & 2.28 & 3.89 & 5.82 & 4.80 \\
\hline & & SEM & 0.29 & 1.35 & 2.09 & 1.95 & 2.45 & 2.03 & 2.06 & 1.10 & 1.45 & 2.49 & 1.91 \\
\hline & \multirow[t]{2}{*}{ Treated } & Mean & 3.06 & 6.63 & 8.03 & 7.24 & 6.60 & 7.41 & 4.96 & 3.49 & 5.57 & 5.97 & 6.76 \\
\hline & & SEM & 1.68 & 3.17 & 2.34 & 1.52 & 1.59 & 1.12 & 0.89 & 0.39 & 1.30 & 2.83 & 2.93 \\
\hline \multirow[t]{4}{*}{ Palmitoyl ethanolamide } & \multirow[t]{2}{*}{ Control } & Mean & 54.7 & 64.7 & 58.3 & 94.7 & 53.5 & 71.9 & 89.9 & 30.8 & 24.7 & 59.3 & 120 \\
\hline & & SEM & 4.70 & 10.3 & 15.3 & 30.5 & 12.7 & 11.2 & 7.80 & 4.70 & 1.50 & 20.8 & 36.3 \\
\hline & \multirow[t]{2}{*}{ Treated } & Mean & 66.2 & 62.5 & 65.6 & 86.1 & 58.6 & 48.4 & 69.1 & 78.8 & 39.5 & 92.0 & 120 \\
\hline & & SEM & 19.2 & 11.9 & 14.7 & 17.3 & 13.9 & 9.20 & 2.40 & 23.7 & 8.3 & 31.5 & 47.9 \\
\hline \multirow[t]{4}{*}{ Oleoyl ethanolamide } & \multirow[t]{2}{*}{ Control } & Mean & 24.2 & 24.3 & 19.0 & 20.3 & 30.5 & 28.5 & 14.0 & 29.1 & 17.6 & 29.3 & 31.0 \\
\hline & & SEM & 4.44 & 1.50 & 2.25 & 2.23 & 1.55 & 2.65 & 1.35 & 5.26 & 4.10 & 4.60 & 2.28 \\
\hline & \multirow[t]{2}{*}{ Treated } & Mean & 24.2 & 22.3 & 16.0 & 20.3 & 24.8 & 29.9 & 14.4 & 36.9 & 18.0 & 39.7 & 45.5 \\
\hline & & SEM & 3.63 & 6.86 & 2.05 & 2.49 & 6.63 & 7.76 & 2.54 & 15.01 & 3.03 & 14.54 & 9.89 \\
\hline \multicolumn{4}{|c|}{ Dose (mg phospholipids/kg bw/day) } & \multicolumn{2}{|l|}{50} & \multicolumn{2}{|l|}{150} & \multicolumn{2}{|l|}{450} & \multicolumn{4}{|l|}{0} \\
\hline Dose $(\mathrm{mg}[\mathrm{EPA} / \mathrm{DHA}] / \mathrm{kg}$ & w/day) & & & $9.35 / 5$ & & $28.0 /$ & & $84.1 / 4$ & & $0 / 0$ & & & \\
\hline
\end{tabular}

Doses of phospholipids and of EPA and DHA are shown at the end of the table

does apparently not alter the ratio of unsaturated vs. saturated FAs but only the relative concentrations of unsaturated FAs. The plasma concentrations of six endocannabinoid-type mediators changed accordingly, as those being downstream arachidonic acid decreased, those downstream the saturated palmitic and oleic acids remained unchanged, and a downstream EPA metabolite increased.

\section{Methods}

\section{Test and control items}

The product tested in the current investigation is a preparation of purified ( $>98 \%$ pure) phospholipids extracted from krill-oil, rich in the long-chain omega-3 fatty acids eicosapentaenoic acid (EPA; C20:5n3) and docosahexaenoic acid (DHA; C22:6n3). The final product was formulated to improve its floating properties, thus making it easier to administer, and had the following composition: phospholipids $84 \%(840 \mathrm{mg} / \mathrm{ml})$, polyethylene glycol with MW 400 (PEG400) 12.5\% and ethanol 3.5\%. The abundance of FAs in the end-product was analyzed by methylation of the FAs followed by gas-chromatography and 25 fatty acids were detected and quantified. The EPA and DHA contents were 15.7\% (157 mg/g) and $9.2 \%(92 \mathrm{mg} / \mathrm{g})$, respectively. In addition, five other FAs were present in the end-product with an abundance of more than $1 \%$ (10 mg/g): myristic acid (C14:0) 1.1\%, palmitic acid (C16:0) $13.2 \%$, oleic acid (C18:1n9) $2.1 \%$, cisvaccenic acid (C18:1n11) $3.1 \%$ and stearidonic acid (C18:4n3) 1.3\%. All other detected FAs in the end-product were below $1 \%$. The formulation used has been proven stable for at least 6 months.

The control substance was designed to be a vehicle control and was made similarly as the test substance but 
by substituting the phospholipids with water. Therefore, the control substance contained $84 \%$ water, $12.5 \%$ PEG400 and 3.5\% ethanol.

\section{Animals}

Six type-2 diabetic and dyslipidemic cynomolgus monkeys (Macaca fascicularis) were selected for the study, after screening a total of 34 animals. Inclusion criteria were predefined and were related to diabetes parameters (HbA1c, glucose and insulin) and blood lipid levels (triglycerides, total cholesterol, low-density lipoprotein cholesterol (LDL-c) and high-density lipoprotein cholesterol (HDL-c)). The results from the screening as well as details of housing conditions and care are described in [11]. Vital data of the monkeys included in the study are given in Table 3.

The animals were fed a standard monkey diet containing crude protein $(\geq 16 \%)$, crude fat $(\geq 4 \%)$, moisture $(\leq 10 \%)$, ash $(\leq 7 \%)$, fiber $(\leq 4 \%)$, calcium $(0.8-1.2 \%)$ and phosphorus $(0.6-0.8 \%)$. The content of omega-3 fatty acids in the diet had not been determined by the supplier (Beijing Keao Xieli Feed Co. Ltd., Beijing, P. R. China). Care and use of the animals were conducted in accordance with all applicable assessment and accreditation of the laboratory animal care (AAALAC) regulations and guidelines. Crown bioscience institutional animal care and use committee (IACUC) approved all animal procedures used in the study. All the procedures related to handling, care and treatment of the animals in this study were performed according to the guidelines approved by AAALAC. After completion of the study the animals were returned to the stock of diabetic monkeys held at Crown Biosciences for a recovery period and following this they were allowed to be used in other studies.

\section{Dosing}

The test and control items were dosed once per day. Just prior to administration the product was warmed up to

Table 3 Vital data of the six non-human primates included in the study

\begin{tabular}{lllll}
\hline Group & Animal ID & Sex & Age (years) & Bodyweight $(\mathrm{kg})$ \\
\hline Control & & & & \\
& Control no. 1 & M & 14 & 9.22 \\
& Control no. 2 & M & 15 & 10.0 \\
& Control no. 3 & F & 13 & 4.32 \\
Treated & & & & \\
& Treated no. 1 & M & 14 & 8.30 \\
& Treated no. 2 & F & 21 & 7.34 \\
& Treated no. 3 & M & 20 & 10.8 \\
\hline
\end{tabular}

$35{ }^{\circ} \mathrm{C}$ and mixed with lukewarm water in a syringe in the ratio 1:5 to become an emulsion. Immediately thereafter, the emulsion was administered by connecting a naso-/oral gastric tube to the syringe and inserting it into the stomach. To ascertain that the entire dose was given the tube was flushed with lukewarm water. The control item was handled in the same way as the test item.

The control and test items were given by gavage, $1 \mathrm{~h}$ after the first feeding of the day. The control article was administered at the same volume $/ \mathrm{kg}$ as the test article. For the first 4 weeks, a phospholipid dose of $50 \mathrm{mg} / \mathrm{kg} /$ day was given, the next 4 weeks the dose was $150 \mathrm{mg} /$ $\mathrm{kg} /$ day and for the last 4 weeks $450 \mathrm{mg} / \mathrm{kg} /$ day. These phospholipid doses resulted in EPA/DHA doses of 9.35/ $5.48 \mathrm{mg} / \mathrm{kg} /$ day, $28.0 / 16.4 \mathrm{mg} / \mathrm{kg} /$ day and $84.1 / 49.3 \mathrm{mg} /$ $\mathrm{kg} /$ day, respectively. Dosing volumes were based on body weights recorded bi-weekly. When converted to human equivalent dose (HED) these doses were equivalent to approximately 1,3 and $10 \mathrm{~g}$ phospholipids/day, respectively, to an adult person of $70 \mathrm{~kg}$.

\section{Blood sampling}

Blood was sampled bi-weekly and centrifuged at 3000$3500 \mathrm{rpm}$ for $10-15 \mathrm{~min}$ at room temperature. After discarding the plasma and buffy coat a minimum of $0.25 \mathrm{ml}$ red blood cells (RBC) from the middle of the RBC layer was collected and transferred to a well-labeled storage cryovial. The samples were frozen at $-80{ }^{\circ} \mathrm{C}$ until being shipped out on dry ice for analysis.

\section{Analysis}

The analysis of fatty acids in the erythrocyte membranes was performed by OmegaQuant, Sioux Falls, SD USA, by gas chromatography $(\mathrm{GC})$ with flame ionization detection [6]. OmegaQuant is a Clinical Laboratory Improvement Amendments (CLIA)-certified laboratory and the assay for fatty acid analysis has been validated and standardized [29]. The company has not published all details of the method and the validation of it since they consider this information proprietary. However, the coefficient of variation $(\mathrm{CV})$ of the method for EPA, DHA and arachidonic acid is reported to be between 5 and $8 \%$ for all three fatty acids [29] and for the omega-3 index the CV is reported to be $<5 \%[6]$.

Unwashed, packed erythrocytes were transferred to a screw-cap glass vial. Methanol containing 14\% boron trifluoride (Sigma-Aldrich, St. Louis, MO) and hexane (EMD Chemicals, USA) were added sequentially. The vial was briefly vortexed and heated in a hot bath at $100{ }^{\circ} \mathrm{C}$ for $10 \mathrm{~min}$. After cooling, HPLC grade water was added, the tubes were recapped, vortexed and centrifuged to separate layers. An aliquot of the hexane layer was transferred to a GC vial. GC was carried out using a 
GC2010 Gas Chromatograph (Shimadzu Corporation, Columbia, MD) equipped with an SP2560, 100-m fused silica capillary column $(0.25 \mathrm{~mm}$ internal diameter, 0.2 um film thickness; Supelco, Bellefonte, PA).

FAs were identified by comparison with a standard mixture of fatty acids characteristic of RBC (GLC OQA, NuCheck Prep, Elysian, MN) which was also used to determine individual fatty acid calibration curves. In addition to EPA (C20:5n3) and DHA (C22:6n3), the following 22 fatty acids were quantified: myristic (14:0), palmitic (16:0), palmitoleic (C16:1n7), palmitelaidic $(\mathrm{C} 16: 1 \mathrm{n} 7 \mathrm{t})$, stearic $(18: 0)$, oleic $(\mathrm{C} 18: 1 \mathrm{n} 9)$, elaidic (C18:1n9t), linoleic (C18:2n6), linoelaidic (C18:2n6t), alpha-linolenic (C18:3n3), gamma-linolenic (C18:3n6), arachidic (20:0), eicosenoic (C20:1n9), eicosadienoic (C20:2n6), dihomo-g-linolenic (C20:3n6), arachidonic (C20:4n6), behenic (22:0), docosatetraenoic (C22:4n6), docosapentaenoic (C22:5n 3 and $\mathrm{C} 22: 5 \mathrm{n} 6)$, lignoceric (24:0) and nervonic (C24:1n9) acids. The chromatographic conditions used in this study were sufficient to isolate the C16:1trans isomers and the C18:2, $9 \mathrm{t}-12 \mathrm{c}$, $9 \mathrm{t}-12 \mathrm{t}$, and $9 \mathrm{c}-12 \mathrm{t}$ isomers, which are reported as C18:2n6t. However, each individual C18:1 trans molecular species (i.e., C18:16 thru 13) could not be segregated but appeared as two blended peaks that eluted just before oleic acid. The areas of these two peaks were summed and referred to a C18:1 trans.

FA composition was expressed as a percent of total identified fatty acids. The omega- 3 index was calculated as the sum of percent fraction of EPA $(\mathrm{C} 20: 5 \mathrm{n} 3)$ and DHA (C22:6n3).

The concentrations of the endocannabinoids anandamide (AEA), 2-arachidonoylglyceride (2-AG), EPA ethanolamide (EPAEA), DHA ethanolamide (DHAEA), palmitoyl ethanolamide (PEA) and oleoyl ethanolamide (OEA) in plasma were analyzed as described previously [30]. In brief, samples were dounce-homogenized and extracted with chloroform/methanol/Tris- $\mathrm{HCl} 50 \mathrm{mM}$ pH $7.5(2: 1: 1, \mathrm{v} / \mathrm{v})$ containing internal deuterated standards for AEA, 2-AG, PEA and OEA quantification by isotope dilution (10 pmol of $\left[{ }^{2} \mathrm{H}\right]_{8} \mathrm{AEA}$ and 50 pmol of $\left[{ }^{2} \mathrm{H}\right]_{5} 2 \mathrm{AG},\left[{ }^{2} \mathrm{H}\right]_{4}$ PEA, $\left[{ }^{2} \mathrm{H}\right]_{4}$ OEA (Cayman Chemicals, MI, USA). Quantification of EPAEA and DHAEA was done by using deuterated AEA as internal standard. The lipid-containing organic phase was dried down, weighed and pre-purified by open bed chromatography on silica gel. Fractions were obtained by eluting the column with 99:1, 90:10 and 50:50 (v/v) chloroform/ methanol. The 90:10 fraction was used for AEA, 2-AG, PEA and OEA quantification by liquid chromatographyatmospheric pressure chemical ionization-mass spectrometry (LC-APCI-MS), as previously described and using selected ion monitoring at $\mathrm{M}+\mathrm{H}^{+}$values for the four compounds and their deuterated homologues, as described in [31, 32]. Pilot experiments showed that the ratios between the peak areas of the $\mathrm{M}+\mathrm{H}^{+}$ions for each of the non-deuterated compounds and those of a known amount (10 or $50 \mathrm{pmol}$ ) of the corresponding deuterated compounds (or of deuterated AEA in the case of EPAEA and DHAEA) varied linearly with the concentration of the non-deuterated compounds, over the $0.1-1000 \mathrm{pmol}$ range, and therefore were suitable for quantification. The $\mathrm{CV}$ of these methods have also been described in previous papers (see [32] and [33] for recent reviews).

\section{Data presentation and statistics}

For the omega-3 FAs EPA and DHA, and for the omega-3 index, individual values for each of the 6 animals are presented graphically. For the other 22 FAs, mean values for the control and treated groups are tabulated and mean and SEM values presented graphically. For the endocannabinoids, mean and SEM values for the control and treated groups are tabulated. Mean rather than median values were used to describe the central tendency for each group, despite the small group size, since this also made it possible to give SEM as a measure of spread. Due to the low number of animals in each of the control and treated groups, no comparison between groups based on statistical criteria was performed.

\section{Abbreviations}

2-AG: 2-Arachidonoylglycerol; ANA: Anandamide; bw: Body weight; DHA: Docosahexaenoic acid; DHAEA: DHA ethanolamide;

EPA: Eicosapentaenoic acid; EPAEA: EPA ethanolamide; FA: Fatty acid; OEA: Oleoyl ethanolamide; PEA: Palmitoyl ethanolamide; RBC: Red blood cells

\section{Acknowledgements}

The authors thank OmegaQuant LLC, SD USA for performing the chemical analyses of the fatty acids in erythrocyte membranes. We also thank Dr. Nils Hoem at Aker Biomarine, Lysaker Norway for reviewing the manuscript and providing useful input to it.

\section{Funding}

This study was funded by Aker Biomarine Antarctic AS.

\section{Availability of data and material}

The datasets generated during the current study are available from the corresponding author on reasonable request.

\section{Authors' contributions}

PAH designed the study, analysed and interpreted the data and wrote the majority of the manuscript. XLW performed the practical part of the experiments and contributed to the manuscript preparation. FB and VD analysed the plasma samples for endocannabinoids and endocannabinoidlike mediators, interpreted the results and contributed to the preparation of the manuscript. YFX designed the study, performed practical parts of the study, organized and interpreted the data and contributed to the preparation of the manuscript. All authors have read and approved the final manuscript.

\section{Competing interests}

PAH is an employee of Aker Biomarine Antarctic AS, Norway; XLW and YFX are employees of Crown BioSciences, Inc., P.R. China. These authors declare that they have no other competing interests. FB declares that she has no competing interests. VD was a member of Aker Biomarine's Scientific Advisory Board until 2015 but declares that he has no other competing interests. 


\section{Consent for publication}

Not applicable

\section{Ethics approval}

The experimental protocol was reviewed and approved by the Institutional Animal Care and Use Committee (IACUC) of Crown Bioscience, Inc., approval no. AN-1308-016-15. During the study, any change of the approved protocol was submitted as an amendment to the IACUC for approval before conducting the changed procedure. Crown Bioscience, Inc. is accredited by the Association for Assessment and Accreditation of Laboratory Animal Care (AAALAC) International.

\section{Author details}

'Aker Biomarine Antarctic AS, Oksenoyveien 10, N-1366 Lysaker, Norway. ${ }^{2}$ Crown Bioscience (Taicang) Inc., Science and Technology Park, 6 Beijing West Road, Taicang, Jiangsu Province, People's Republic of China. ${ }^{3}$ Endocannabinoid Research Group, Institute of Biomolecular Chemistry, Consiglio Nazionale delle Ricerche, Pozzuoli, NA, Italy.

\section{Received: 6 October 2016 Accepted: 11 January 2017}

\section{Published online: 21 January 2017}

\section{References}

1. Calder P. Very long chain omega-3 (n-3) fatty acids and human health. Eur J Lipid Sci Tech. 2014;116:1280-300.

2. Tou JC, Jaczynski J, Chen YC. Krill for human consumption: nutritional value and potential health benefits. Nutr Rev. 2007;65:63-77.

3. Pedersen AM, Vang B, Olsen RL. Oil from Calanus finmarchicus-composition and possible use: a review. J Aquat Food Prod Technol. 2014:23:633-46.

4. Bernstein AM, Ding EL, Willett WC, Rimm EB. A meta-analysis shows that docosahexaenoic acid from algal oil reduces serum triglycerides and increases HDL-cholesterol and LDL-cholesterol in persons without coronary heart disease. J Nutr. 2012;142:99-104.

5. Harris WS, Von Schacky C. The Omega-3 index: a new risk factor for death from coronary heart disease? Prev Med. 2004:39:212-20.

6. Harris WS, Von Schacky C, Park Y. Standardizing methods for assessing omega-3 fatty acid biostatus. In McNamara RK, editor. The Omega-3 fatty acid deficiency syndrome. New York: Nova Science Publishers Inc; 2013. p. 385-98.

7. Naughton SS, Mathai ML, Hryciw DH, McAinch AJ. Fatty acid modulation of the endocannabinoid system and the effect on food intake and metabolism. Int J Endocrinol. 2013:2013:361895.

8. Berge K, Piscitelli F, Hoem N, Silvestri C, Meyer I, Banni S, Di Marzo V. Chronic treatment with krill powder reduces plasma triglyceride and anandamide levels in mildly obese men. Lipids Health Dis. 2013;12:78

9. Piscitelli F, Carta G, Bisogno T, Murru E, Cordeddu L, Berge K, Tandy S, Cohn JS, Griinari M, Banni S, Di Marzo V. Effect of dietary krill oil supplementation on the endocannabinoidome of metabolically relevant tissues from highfat-fed mice. Nutr Metab (Lond). 2011;8:51.

10. Di Marzo V. Targeting the endocannabinoid system: to enhance or reduce? Nat Rev Drug Discov. 2008;7:438-55.

11. Hals PA, Wang X, Xiao YF. Effects of a purified krill oil phospholipid rich in long-chain omega-3 fatty acids on cardiovascular disease risk factors in nonhuman primates with naturally occurring diabetes type-2 and dyslipidemia. Lipids Health Dis. 2017;16:11.

12. Nair $A B$, Jacob $S$. A simple practice guide for dose conversion between animals and human. J Basic Clin Pharm. 2016;7:27-31.

13. Katan MB, Deslypere JP, van Birgelen AP, Penders M, Zegwaard M. Kinetics of the incorporation of dietary fatty acids into serum cholesteryl esters, erythrocyte membranes, and adipose tissue: an 18-month controlled study. J Lipid Res. 1997;38:2012-22.

14. Ramprasath VR, Eyal I, Zchut S, Jones PJ. Enhanced increase of omega-3 index in healthy individuals with response to 4-week n-3 fatty acid supplementation from krill oil versus fish oil. Lipids Health Dis. 2013;12:178.

15. Hulbert AJ, Turner N, Storlien LH, Else PL. Dietary fats and membrane function: implications for metabolism and disease. Biol Rev Camb Philos Soc. 2005;80:155-69.

16. Silvestri C, Di Marzo V. The endocannabinoid system in energy homeostasis and the etiopathology of metabolic disorders. Cell Metab. 2013;17:475-90.
17. Meijerink J, Balvers M, Witkamp R. N-acyl amines of docosahexaenoic acid and other n-3 polyunsatured fatty acids - from fishy endocannabinoids to potential leads. Br J Pharmacol. 2013;169:772-83.

18. Calder PC. n-3 polyunsaturated fatty acids, inflammation, and inflammatory diseases. Am J Clin Nutr. 2006;83:1505S-19.

19. Shinohara M, Serhan CN. Novel endogenous proresolving molecules. J Atheroscler Thromb. 2016;23:655-64.

20. Yurko-Mauro K, Kralovec J, Bailey-Hall E, Smeberg V, Stark JG, Salem Jr N. Similar eicosapentaenoic acid and docosahexaenoic acid plasma levels achieved with fish oil or krill oil in a randomized double-blind four-week bioavailability study. Lipids Health Dis. 2015:14:99.

21. Ulven SM, Kirkhus B, Lamglait A, Basu S, Elind E, Haider T, Berge K, Vik H, Pedersen JI. Metabolic effects of krill oil are essentially similar to those of fish oil but at lower dose of EPA and DHA, in healthy volunteers. Lipids. 2011:46:37-46.

22. Schuchardt JP, Schneider I, Meyer H, Neubronner J, von Schacky C, Hahn A. Incorporation of EPA and DHA into plasma phospholipids in response to different omega-3 fatty acid formulations-a comparative bioavailability study of fish oil vs. krill oil. Lipids Health Dis. 2011;10:145.

23. Iqbal J, Hussain MM. Intestinal lipid absorption. Am J Physiol Endocrinol Metab. 2009;296:E1183-94.

24. Ramprasath VR, Eyal I, Zchut S, Shafat I, Jones PJ. Supplementation of krill oil with high phospholipid content increases sum of EPA and DHA in erythrocytes compared with low phospholipid krill oil. Lipids Health Dis. 2015;14:142.

25. Harris WS, Sands SA, Windsor SL, Ali HA, Stevens TL, Magalski A, Porter CB, Borkon AM. Omega-3 fatty acids in cardiac biopsies from heart transplantation patients: correlation with erythrocytes and response to supplementation. Circulation. 2004;110:1645-9.

26. Harris WS. The omega-3 index as a risk factor for coronary heart disease. Am J Clin Nutr. 2008:87:1997S-2002.

27. Edwards R, Peet M, Shay J, Horrobin D. Omega-3 polyunsaturated fatty acid levels in the diet and in red blood cell membranes of depressed patients. J Affect Disord. 1998;48:149-55.

28. Witte AV, Kerti L, Hermannstadter HM, Fiebach JB, Schreiber SJ, Schuchardt JP, Hahn A, Floel A. Long-chain omega-3 fatty acids improve brain function and structure in older adults. Cereb Cortex. 2013;24:3059-68.

29. OmegaQuant: OmegaQuant methodological considerations. http:// omegaquant.com/methods/. Accessed 10 Dec 2016.

30. Matias I, Carta G, Murru E, Petrosino S, Banni S, Di Marzo V. Effect of polyunsaturated fatty acids on endocannabinoid and $\mathrm{N}$-acyl-ethanolamine levels in mouse adipocytes. Biochim Biophys Acta. 1781;2008:52-60.

31. Di Marzo V, Goparaju SK, Wang L, Liu J, Batkai S, Jarai Z, Fezza F, Miura Gl, Palmiter RD, Sugiura T, Kunos G. Leptin-regulated endocannabinoids are involved in maintaining food intake. Nature. 2001;410:822-5.

32. Bisogno T, Piscitelli F, Di Marzo V. Lipidomic methodologies applicable to the study of endocannabinoids and related compounds: Endocannabinoidomics. Eur J Lipid Sci Technol. 2009:111:53-63.

33. Witkamp RF, Balvers MG. Analysis of Omega-3 fatty acid derived NAcylethanolamines in biological matrices. Methods Mol Biol. 2016;1412:27-40

\section{Submit your next manuscript to BioMed Central and we will help you at every step:}

- We accept pre-submission inquiries

- Our selector tool helps you to find the most relevant journal

- We provide round the clock customer support

- Convenient online submission

- Thorough peer review

- Inclusion in PubMed and all major indexing services

- Maximum visibility for your research

Submit your manuscript at www.biomedcentral.com/submit 